\title{
Abundancia relativa de postlarvas de camarones peneidos en la bahía Salinas del Marqués, Golfo de Tehuantepec, México. Marzo a junio de 1999
}

\author{
Relative abundance of penaeid shrimp postlarvae in the Salinas del Marques Bay, \\ Gulf of Tehuantepec, Mexico. March to June of 1999 \\ Sebastián Ramos-Cruz ${ }^{1}$ y Eduardo Ramos Santiago ${ }^{1}$ \\ ${ }^{1}$ Programa Camarón del Instituto Nacional de la Pesca,Centro Regional de Investigación Pesquera (CRIP) Salina Cruz Prol. Playa \\ Abierta s/n, Col. San Juan Miramar, C.P. 70600 Salina Cruz, Oaxaca, México \\ ramoscruz@yahoo.com
}

\begin{abstract}
From March to June 1999 biweekly sampling of shrimp postlarvae were carried out in the front of the beach of the Salinas del Marques Bay, Oaxaca, Mexico, to evaluate their incidence toward the coastal zone and analyze their possible relationship with the lunar periods and the time of collection. A total of 2,820 postlarvae were collected, of which $L$. vannamei constituted the $87 \%$ followed by $F$. californiensis with the $6.6 \%, F$. brevirostris with the $5.1 \%$ and L. stylirostris with the $1.4 \%$. Three pulses in the postlarval
\end{abstract}

recruitment were identified; the most important had a density of 7 postlarvae $10 \mathrm{~m}^{-3}$. These pulses were more coincident with periods of full moon and the relative abundance diminished from the 06:00 $\mathrm{h}$ to 13:00 $\mathrm{h}$ and increased from 14 $\mathrm{h}$ ahead. These results suggest the probability that the density increases during night hours.

Key words: Crustacea, L. vannamei, L. stylirostris, F. californiensis, F. brevirostris

\section{Introducción}

De los recursos explotados comercialmente en el Golfo de Tehuantepec (GT) y sistemas lagunares adyacentes, el camarón constituye la especie a la que se le dedica el mayor esfuerzo pesquero. El recurso está compuesto por cuatro especies de peneidos: dos del género Litopenaeus (camarón blanco $L$. vannamei y camarón azul $L$. stylirostris) y dos del género Farfantepenaeus (camarón café $F$. californiensis y camarón cristal $F$. brevirostris). El ciclo de vida de las dos primeras especies está estrechamente ligado a la zona costera, pero de manera específica, $L$. vannamei es altamente dependiente de los ambientes estuarinos o lagunares, a los que ingresa en fase postlarval y permanece en su interior hasta alcanzar la fase pre-adulta cuando sale de ellos y se incorpora a la población marina en donde se reproduce; mientras que el ciclo de las dos especies restantes (camarón café y cristal) transcurre en su mayor parte en el área marina. Sin embargo, dado que el proceso reproductivo de las cuatro especies ocurre en el mar y los productos son transportados por las corrientes marinas hacia la costa en donde pueden ser recolectados, identificados y cuantificados, es posible entonces conocer el o los tiempos en los que la abundancia de cada especie es mayor. Entre las investigaciones que abordan aspectos relacionados con las fases larvarias del camarón, pueden citarse las de Pérez et al. (1991), quienes realizaron un estudio sobre los diferentes aspectos del recurso camarón en el sistema lagunar Mar Muerto, OaxacaChiapas, México, detectando que los principales pulsos de reclutamiento se presentan en enero, marzo y junio. En este mismo sistema lagunar, Mariano (1994) analizó la incidencia postlarval concluyendo que la especie dominante fue $L$. vannamei, la que mostró su mayor incidencia durante la noche y la madrugada. MedinaReyna \& Salinas (1996), concluyeron que en la bahía La Ventosa, Oaxaca, México, la especie dominante fue $F$. californiensis (camarón café), seguida por $F$. brevirostris (camarón cristal) y, en tercer lugar, por $L$. vannamei (camarón blanco). Macías-Regalado et al. (1982) analizaron los factores ambientales que influyen en los movimientos migratorios de las postlarvas hacia las lagunas costeras de Sinaloa, México, concluyendo que las corrientes de agua constituyen uno de los factores que determinan dichos movimientos hacia los sistemas lagunares. Calderón-Pérez \& Poli (1987) concluyeron que la migración de postlarvas hacia la costa está en función de la dirección del viento y 
corrientes de deriva. Sánchez-Meráz \& Martínez-Vega (2000) analizaron la inmigración de postlarvas hacia un sistema lagunar de Oaxaca, México, concluyendo que el ingreso de las mismas está condicionada por la intensidad del flujo de marea y las condiciones hidrológicas imperantes en la boca del sistema lagunar.

Conocer la abundancia y las tallas de las postlarvas en el tiempo permite incrementar el conocimiento sobre los aspectos reproductivos y de crecimiento de las especies involucradas, para fortalecer los esquemas de manejo del recurso, principalmente de aquellas especies que son sujetas a explotación con fines de acuicultura. Bajo esta premisa, en el presente documento se analizan las abundancias relativas de postlarvas de camarón y su relación con los efectos lunares y la hora de recolecta, así como las tallas medias que tienen los organismos al momento de arribar a la costa. Conscientes de que el período de estudio es corto y consecuentemente las observaciones derivadas del mismo no proporcionan un panorama mucho más amplio del proceso de reclutamiento postlarval y los factores que influyen sobre el mismo hacia el área de estudio, se ha considerado oportuno dar a conocer estos resultados con la finalidad de que puedan servir como referencia a futuras investigaciones que se lleven a cabo en la misma zona de estudio o en áreas circunvecinas.

\section{Area de estudio}

La bahía Salinas del Marqués forma parte del Golfo de Tehuantepec (GT) (Fig. 1) y se localiza frente a la comunidad del mismo nombre, a escasos cuatro kilómetros hacia el oeste de la ciudad y puerto de Salina Cruz, Oaxaca, México (Gracia et al. 1986). Estos autores mencionan que la bahía presenta una profundidad media de $30 \mathrm{~m}$ con sedimentos lodosos, que su margen litoral presenta una pendiente suave hacia el fondo y sus playas están constituidas por arena fina, presentando un relieve suave casi plano. Frente a la bahía se localiza, un pequeño cuerpo de agua salobre conocido como "Las Salinas" de donde se extrae sal en período de secas y se captura camarón durante la temporada lluviosa; su longitud es de aproximadamente $2 \mathrm{~km}$ con una amplitud media de $150 \mathrm{~m}$. Su profundidad media es de $0,70 \mathrm{~m}$ y se conecta con la bahía a través de un canal artificial de aproximadamente $80 \mathrm{~m}$ de longitud, por donde penetran las postlarvas durante el rebalsamiento de las mareas, las cuales son de tipo mixto.

El área de estudio se localiza dentro de la región climática del tipo Aw”, que corresponde a un clima cálido subhúmedo, el más seco de los subhúmedos, con lluvias en verano y presencia de Canícula e Isoterma (García 1981), con una marcha anual de la temperatura que lo sitúa dentro del tipo Ganges (Anónimo 1988). La temperatura promedio del agua superficial del GT varía entre 25 y $30^{\circ} \mathrm{C}$ (Gallegos-García \& Barberán-Falcón 1998). Los vientos dominantes son los “nortes" o “tehuanos” (Medina-Reyna 1999), los cuales vienen del norte, aumentando su notoriedad de octubre a abril, con velocidades promedio de 27 km/h (Monreal-Gómez \& Salas de León 1998).

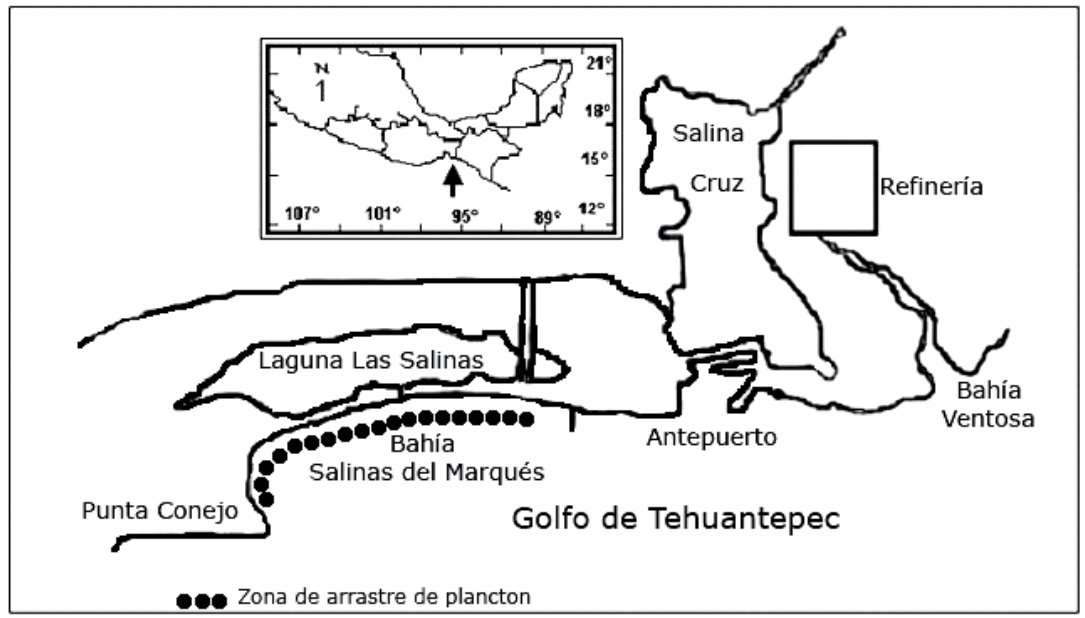

Figura 1

Localización del área de estudio en el Golfo de Tehuantepec, México

Study area at the Gulf of Tehuantepec, Mexico 


\section{Material y métodos}

Se realizaron muestreos quincenales de marzo a junio de 1999, en períodos de influencia lunar (luna nueva y llena). Los arrastres se efectuaron manualmente y al nivel superficial en el frente de playa, sobre un transecto paralelo a la línea de costa, efectuándose el arrastre en contra de la dirección predominante del oleaje, a una profundidad promedio de 1,5 m. La duración de cada arrastre fue de 5 minutos a intervalos de una hora, abarcando el período de iluminación natural (06:00 h a 18:00 h), sin considerar el tipo de marea presente en ese intervalo, esto es, los muestreos se realizaron de manera continua desde las 06:00 h hasta las 18:00 h. Se utilizó una red cónica de plancton de $30 \mathrm{~cm}$ de diámetro por 1,5 m de alto y abertura de malla de $500 \mu \mathrm{m}$, equipada con un contador de flujo. Los organismos recolectados fueron conservados en una solución preparada a base de formol-alcohol-ácido acético en una proporción de 3560-5\%, diluida en agua de mar al 20\%. En el laboratorio, las postlarvas fueron separadas e identificadas a nivel de especie (Mair 1979, CalderónPérez et al. 1989). Posteriormente, a cada postlarva se le registró la longitud total en $\mathrm{mm}$, considerada desde la punta del rostro hasta el extremo del telson, realizándose las mediciones con papel milimétrico sujeto a una caja de petri. La concentración de postlarvas fue considerada como la captura estandarizada por cada $10 \mathrm{~m}^{3}$ de agua filtrada, de acuerdo con la siguiente expresión: $(\mathrm{N} * 10)$ / V, donde $\mathrm{N}$ = número de postlarvas capturadas en cada arrastre $\mathrm{y}$ $\mathrm{V}=$ volumen filtrado por la red. Adicionalmente, en cada muestreo se registró la temperatura del agua superficial con un termómetro de mercurio de inmersión total con escala mínima de $0,1^{\circ} \mathrm{C}$, así como la salinidad superficial con un refractómetro óptico. Generalmente, la distribución de las postlarvas en el medio natural no es uniforme sino que se presenta en parches (SánchezMeráz \& Martínez-Vega 2000), por lo que frecuentemente genera valores bajos y otros muy altos, haciendo que los datos no se apeguen a una distribución normal. Bajo esta circunstancia, los registros de abundancia fueron transformados a logaritmos naturales para que los valores resultantes pudieran ser analizados mediante técnicas de análisis estándar. Se efectuaron análisis estadísticos (ANOVA) (Mason \& Lind 1998) para detectar diferencias significativas entre la abundancia postlarval con las fases lunares y la hora de recolecta.

\section{Resultados}

\section{Temperatura $\left({ }^{\circ} \mathrm{C}\right.$ ) y salinidad (psu)}

Al término de los muestreos se obtuvieron 104 registros de temperatura y salinidad superficiales. En el caso de la temperatura, los valores fluctuaron entre 21 y $32^{\circ} \mathrm{C}$, con valor promedio de $28,3^{\circ} \mathrm{C} \pm 0,29$ D.E. En tanto que los registros de salinidad variaron entre 30 y 45 psu, situándose la salinidad promedio en 36,1 \pm 0,33 D.E. Al agrupar los registros por períodos de muestreo y obtener sus valores medios (Fig. 2), se observó en el caso de la temperatura que los valores promedio variaron entre 22,2 y $31,3^{\circ} \mathrm{C}$ (Fig. 2), mientras que los de la salinidad se ubicaron entre 31,3 y 40 psu, exhibiendo ambos parámetros un patrón inverso respecto uno del otro (Fig. 2).

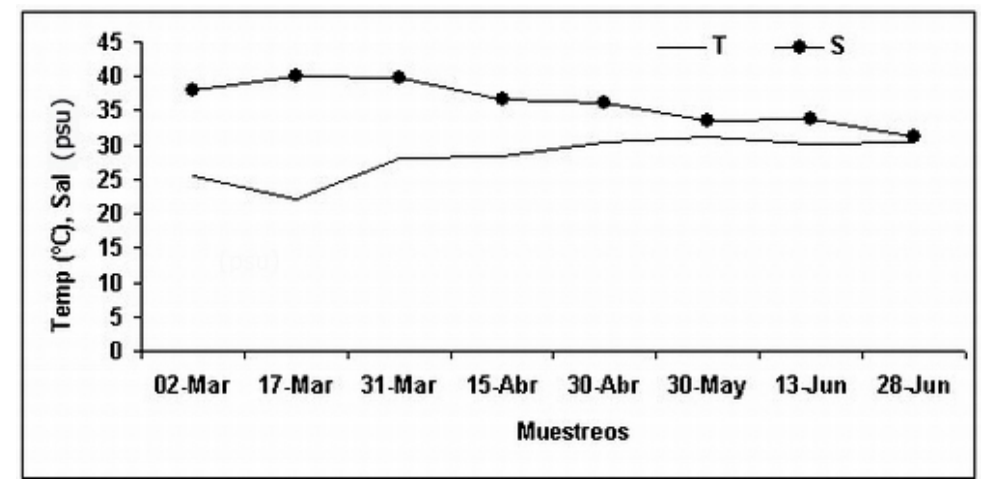

Figura 2

Tendencia de los valores promedio de temperatura $\left({ }^{\circ} \mathrm{C}\right)$ y salinidad (psu) del agua superficial en la bahía Salinas del Marqués, de marzo a junio de 1999

Tendency of the average temperature $\left({ }^{\circ} \mathrm{C}\right.$ ), and salinity (psu) values of the surface water in the Salinas del Marques Bay, from March to June 1999 


\section{Composición de especies}

Al finalizar el período de muestreo se recolectaron 2.820 postlarvas de camarón, fluctuando las capturas entre 26 y 893 organismos/campaña, con un promedio de 352,5 organismos/campaña. Del total recolectado, $L$. vannamei representó el $87 \%$, seguida por $F$. californiensis el $6,6 \%, F$. brevirostris con el 5,1\% y $L$. stylirostris con el $1,4 \%$.

\section{Abundancia postlarval}

Para todas las especies se identificaron tres pulsos principales en el reclutamiento postlarval al área de estudio; los dos primeros se presentaron durante las primeras quincenas de marzo y abril, respectivamente, con abundancias similares de 3 postlarvas por cada 10 $\mathrm{m}^{3}$, y el tercer pulso se hizo evidente durante la segunda quincena de junio con 7 postlarvas por cada $10 \mathrm{~m}^{3}$. Al analizar estas abundancias por especie y su relación con la hora de recolecta, se observó que $L$. vannamei fue más abundante entre las 10:00 h y las 13:00 h, y de las 14:00 a las 18:00 h. Sin embargo, estas diferencias en las abundancias no fueron evidenciadas estadísticamente $(F=1,4402$, df $=12,13, P>0,05)$ (Tabla 1). En tanto que $F$. californiensis se mantuvo con bajos niveles de abundancia entre las 06:00 $\mathrm{h}$ y las 12:00 h, incrementándose gradualmente a partir de las
14:00 h; no obstante, las diferencias encontradas tampoco fueron estadísticamente significativas $(F=$ 0,4835, df $=12,13, P>0,05)$. En el caso de $L$. stylirostris se detectaron tres períodos de abundancia: los dos primeros de baja intensidad se presentaron entre las 07:00 y las 08:00 h, y a las 12:00 h, en tanto que el tercero y más importante se observó entre las 14:00 y 18:00 h. No obstante, estas diferencias no fueron significativas $(F=0,8488, \mathrm{df}=7,8, \quad P>0,05)$. Finalmente, en $F$. brevirostris se identificaron tres pulsos: el primero entre las 06:00 h y las 09:00 h, el segundo entre las 11:00 h y las 12:00 h, y el tercero entre las 14:00 h y las 18:00 h. Estas abundancias tampoco fueron validadas por el análisis de varianza $(F=0,8561, \mathrm{df}=12,13, P>0,05)$ (Tabla 1$)$.

Respecto a las abundancias y su relación con los efectos lunares, el $18 \%$ de las postlarvas se capturó en período de luna nueva y el $82 \%$ con luna llena. Al contrastar las abundancias con los respectivos periodos lunares por medio del ANOVA, los resultados revelaron diferencias significativas para $F$. brevirostris $(F=$ $6,1435$, $\mathrm{df}=1,2, P<0,05)$ y para $L$. vannamei $(F=$ $5,9569$, df $=1,2, P<0,05)$. Mientras que para $L$. stylirostris $(F=0,5140, \mathrm{df}=1,2, P>0,05)$ y $F$. californiensis $(F=0.0939, \quad \mathrm{df}=1,2, \quad P>0,05)$ estadísticamente las abundancias no mostraron una relación significativa con el efecto lunar (Tabla 1).

Tabla 1

Resultados del análisis de varianza entre la abundancia de postlarvas, la hora de muestreo y las fases lunares (luna nueva, luna llena)

Results of analysis of variance among the postlarvae abundance, the collection time and the moon phases (new moon, full moon)

\begin{tabular}{cccccc}
\hline Especie & SC & GL & CM & F & P \\
& & \multicolumn{3}{c}{ Abundancia - hora de muestreo } \\
\hline F. brevirostris & 4,4455 & 12 & 0,3705 & 0,8561 & 0,5965 \\
F. californiensis & 3,0498 & 12 & 0,2542 & 0,4835 & 0,9069 \\
L. stylirostris & 0,9343 & 7 & 0,1335 & 0,8488 & 0,5791 \\
L. vannamei & 26,3517 & 12 & 2,1960 & 0,1702 \\
& & & Abundancia - efecto lunar & & \\
\hline F. brevirostris & 2,2710 & 1 & 2,2710 & 0,1435 & 0,0174 \\
F. californiensis & 0,0425 & 1 & 0,0425 & 0,0939 & 0,7610 \\
L. stylirostris & 0,0776 & 1 & 0,0776 & 0,5140 & 0,4852 \\
L. vannamei & 9,1183 & 1 & 9,1183 & 5,9569 & 0,0169 \\
\hline
\end{tabular}

SC = Suma de Cuadrados, GL = Grados de Libertad, $\mathbf{C M}=$ Cuadrado Medio, $\mathbf{F}=$ Valor Crítico del estadístico $F$ y $\mathbf{P}=$ Probabilidad al $95 \%$ 

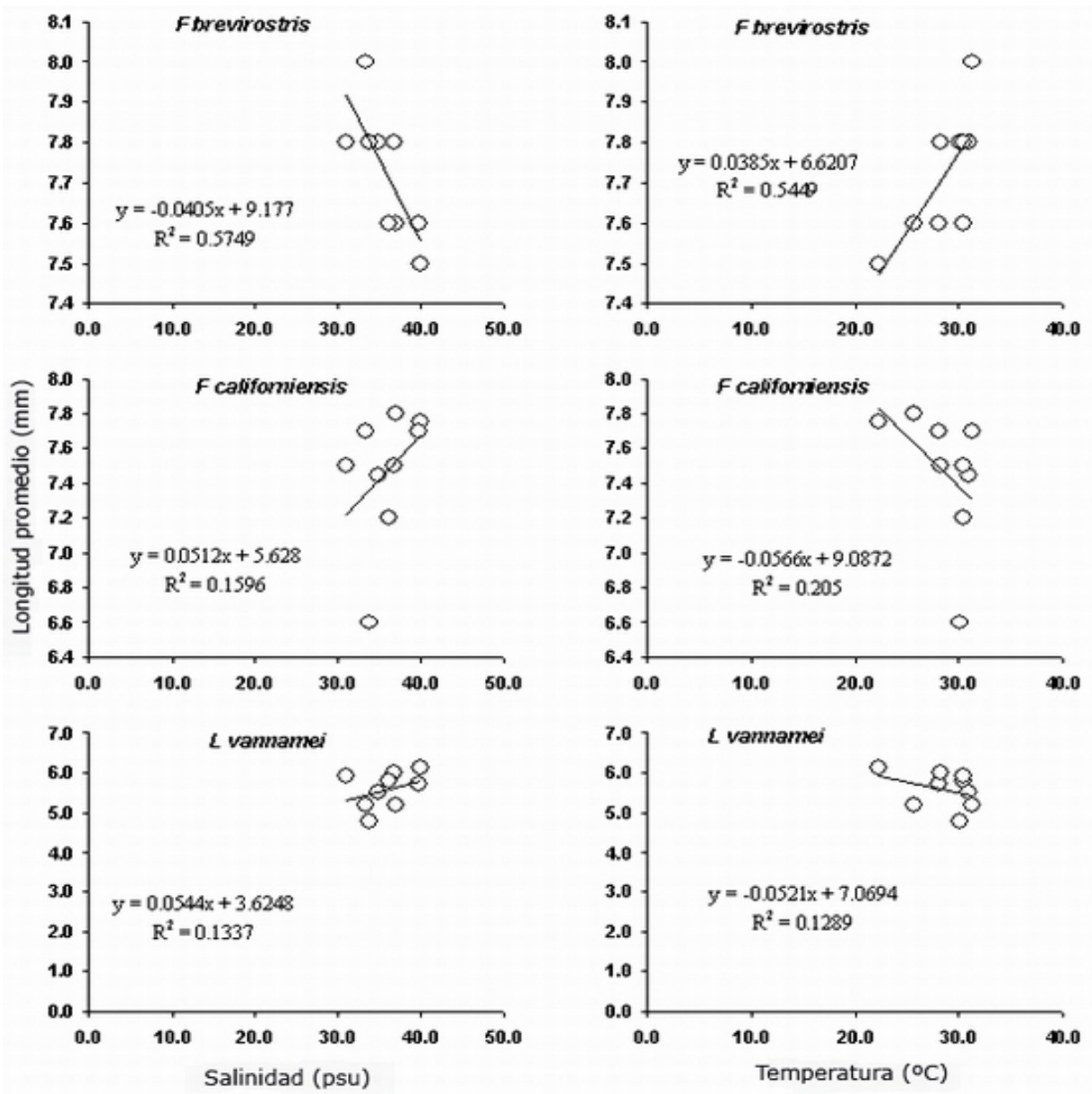

Figura 3

Relación entre la talla media $(\mathrm{mm})$ postlarval de camarones peneidos, la salinidad (psu) y la temperatura $\left({ }^{\circ} \mathrm{C}\right)$ superficiales, en la bahía Salinas del Marqués, de marzo a junio de 1999

Relationship between the mean size (mm) of peneid shrimp postlarvae, the surface water salinity (psu) and temperature $\left({ }^{\circ} \mathrm{C}\right)$ in the Salinas del Marques Bay, from March to June 1999

Respecto a las abundancias y su relación con los efectos lunares, el $18 \%$ de las postlarvas se capturó en período de luna nueva y el $82 \%$ con luna llena. Al contrastar las abundancias en relación con los efectos lunares por medio del ANOVA, los resultados revelaron diferencias significativas para $L$. stylirostris $(F=$ $0,5140, \mathrm{df}=1,2, P<0,05)($ Tabla 1$)$ y $L$. vannamei $(F=$ 5,9569, df $=1,2, P<0,05)$, mientras que para $F$. californiensis y $F$. brevirostris las diferencia no fueron significativas $(P>0,05)$ (Tabla 1$)$.

\section{Estructura por tallas}

La talla promedio de F. brevirostris fluctuó entre 7,5 y
$8,0 \mathrm{~mm}$, en tanto que $F$. californiensis exhibió tallas promedio de 6,6 a 7,8 $\mathrm{mm}$. En cambio, las tallas de $L$. vannamei fluctuaron entre 4,8 y 6,1 mm, mientras que L. stylirostris exhibió una talla promedio de $7,0 \mathrm{~mm}$. Al analizar la posible relación entre las tallas medias y los valores medios de temperatura y salinidad, se observó que las tallas de $F$. brevirostris se agruparon en el intervalo de 21,5 a $31,3^{\circ} \mathrm{C}$ y entre los 31,1 a $40 \mathrm{psu}$, mientras que las de $F$. californiensis se concentraron en el intervalo de los 26 a $31,5^{\circ} \mathrm{C}$ y entre los 30,8 y 39,7 psu. En tanto que las tallas de L. vannamei se agruparon alrededor de los 22,3 y $31,3^{\circ} \mathrm{C}$, y entre los 31,1 y 40 psu (Fig. 3). 


\section{Discusión}

Durante el período de muestreo, la temperatura y la salinidad superficiales mostraron una tendencia inversa que reflejó las condiciones hidrográficas de la bahía en el lapso estudiado, observándose una mayor variabilidad en los promedios de la temperatura. Esta fluctuación, sobre todo la acontecida en marzo, está asociada con la presencia de los vientos “norte”, cuyas velocidades promediaron los $34 \mathrm{~km} / \mathrm{h}$ lo que dificultó en gran parte las actividades de muestreo y también constituyó uno de los factores que influyó sobre los resultados finales.

Respecto a la composición de especies, los resultados revelaron que en el área prospectada la población postlarval de camarón estuvo predominantemente integrada por $L$. vannamei, que llegó a constituir hasta el $87 \%$ de las muestras recolectadas, seguida por $F$. californiensis que representó el 6,6\%. Mientras que $F$. brevirostris y $L$. stylirostris representaron de manera conjunta el 6,5\%. En primera instancia esta composición porcentual no es casual ni puntual, ya que históricamente ambas especies, camarón café y camarón blanco, han constituido más del $80 \%$ de las capturas de camarón que se obtienen en el Golfo de Tehuantepec, aunque en términos de biomasa ha dominando en la proporción el camarón café sobre el camarón blanco. Durante la temporada 2000-2001, ambas especies representaron el 84,7\% (46,1\% camarón blanco y 38,6\% camarón café) y de septiembre de 2005 a enero de 2006 (temporada de pesca 2005-2006), las capturas acumuladas estuvieron integradas en un $81,3 \%$ de camarón café $(50,7 \%)$ y camarón blanco $(30,6 \%)^{1}$. Sin embargo, estos resultados difieren de los obtenidos por Medina-Reyna \& Salinas (1996) en la bahía La Ventosa, distante aproximadamente $10 \mathrm{~km}$ del área de estudio y sobre la misma línea de costa, en donde la especie dominante fue $F$. californiensis con el $53,8 \%$ seguida por $F$. brevirostris con el 24,1\% y L. vannamei con el 22,1\%. En cambio son coincidentes con los resultados proporcionados por Mariano (1994) para la boca del Mar Muerto, Chiapas, México, en donde la composición

\footnotetext{
1 Ramos-Cruz, S. Análisis fundamentado para el cierre de la temporada de pesca del camarón 2005-2006, e inicio del período de veda 2006 en la zona marina del Golfo de Tehuantepec, México. Marzo, 2006. Dictamen técnico. Centro Regional de Investigación Pesquera DGIPPS. Instituto Nacional de Pesca. SAGARPA, México. $14 \mathrm{pp}$.
}

postlarval estuvo dominada por el camarón blanco (77,5\%) y el camarón café (21,8\%). En tanto que Sánchez-Meráz \& Martínez-Vega (2000) señalan que en la Boca El Oro del Sistema Lagunar CorraleroAlotengo, Oaxaca, México, los valores más altos de incidencia postlarval correspondieron a $F$. brevirostris seguida por F. californiensis, mientras que $L$. stylirostris y L. vannamei fueron las menos abundantes.

Estas diferencias pueden ser explicadas con base en el diseño de muestreo, esto es, los resultados aquí expuestos corresponden únicamente a muestreos efectuados durante el día, mientras que los resultados publicados en la literatura citada corresponden a ciclos de 24 horas, coincidiendo en que las principales abundancias se presentan durante los períodos de oscuridad. Medina-Reyna \& Salinas (1996) observaron que las principales abundancias de $F$. californiensis se presentaron entre las 21:00 h y las 04:00 h, en tanto que L. vannamei fue más abundante entre las 22:00 h y las 05:00 h. Mientras que los resultados expuestos por Mariano (1994) revelan que $F$. californiensis fue más abundante entre las 21:00 h y las 04:00 h, en tanto que L. vannamei incrementó sustancialmente su abundancia entre las 22:00 h y las 03:00 h. Por su parte SánchezMeráz \& Martínez-Vega (2000) mencionan que las mayores abundancias de postlarvas en el estrato superficial se presentaron durante la noche. En tanto que Macías-Regalado et al. (1982) mencionan que el efecto de la luz sobre los movimientos migratorios de las postlarvas no lo pudieron evaluar; sin embargo, observaron que la mayor abundancia relativa de postlarvas se manifestó durante el período de lluvias cuando la penetración de la luz es inhibida por la gran cantidad de sedimentos en suspensión. Finalmente, García \& Le Reste (1986) mencionan que el tema de la abundancia postlarval y su relación con el ritmo nictimeral ha sido abordado en diversas investigaciones, concluyendo que los resultados no han sido coherentes dada la disimilitud de los mismos.

Por otro lado, al relacionar las abundancias postlarvarias de las cuatro especies con la hora de recolecta, los resultados no revelaron una estrecha relación entre ambas variables, lo que fue validado por el análisis de varianza al no revelar diferencias significativas $(P>0,05)$. En tanto que al analizar las abundancias en relación con el período lunar (luna nueva y luna llena), los resultados fueron más coincidentes con los períodos de luna llena, cuando se obtuvo el $82 \%$ del total de las postlarvas recolectadas. De acuerdo con el ANOVA, esta relación resultó 
estadísticamente significativa para $L$ vannamei y $F$. brevirostris $(P<0,05)$, mientras que para $F$. californiensis y L. stylirostris las diferencias no fueron significativas $(P>0,05)$, denotando una relación independiente entre ambos eventos. Estos resultados son coincidentes con los obtenidos por Medina-Reyna \& Salinas (1996) y Sánchez-Meráz \& Martínez-Vega (2000), quienes observaron que las principales abundancias de postlarvas se presentaron durante el período de luna llena. Por su parte, Mariano (1994) concluye que la abundancia postlarval no mostró diferencias importantes respecto a las fases lunares. Finalmente, García \& Le Reste (1986) mencionan que generalmente las capturas de postlarvas se incrementan durante los períodos de luna nueva, aunque también se han reportado pulsos importantes en la fase de luna llena.

Se observó una aparente relación entre las tallas medias de $F$. brevirostris, $F$. californiensis y $L$. vannamei con la temperatura y la salinidad (Fig. 3). Sin embargo, un análisis de correlación reveló una relación moderada $\left(r^{2}=0,5\right)$ entre la temperatura, la salinidad y las tallas medias para $F$. brevirostris, mientras que para las dos especies restantes la relación resultó muy débil $\left(r^{2}<0,2\right)$. Aún cuando estadísticamente la relación entre las variables involucradas resultó ligera, existen evidencias de que ambas variables físicas juegan un papel importante en la biología de estas especies, sobre todo la salinidad. Al respecto, Macías-Regalado \& Calderón-Pérez (1979) observaron una aparente relación entre la talla media de inmigración de las postlarvas con los valores de salinidad y temperatura, obteniendo una correlación positiva entre la salinidad y la longitud promedio, siendo inversa entre la talla media y la temperatura. Webb \& Kneib (2004) observaron una mayor correlación entre la tasa media de crecimiento de Litopenaeus setiferus con la disminución de la salinidad por el aporte de agua dulce al sistema estuarino, mencionando también que la temperatura puede afectar la tasa de crecimiento y la distribución de los juveniles en los estuarios.

Dada la importancia que tienen estos estudios en el entendimiento del ciclo biológico de las especies que integran el recurso y que permiten el diseño de esquemas más adecuados para su aprovechamiento pesquero, se hace necesario retomar e intensificar las investigaciones al respecto, toda vez que en las cercanías del área de estudio existen algunos sistemas lagunares de pequeñas dimensiones que pueden verse beneficiados con un esquema de repoblamiento postlarval, con lo que seguramente sus producciones anuales se verían incrementadas.

\section{Agradecimientos}

A E. Alberto Aragón Noriega, Daniel Hernández Montaño y a los revisores anónimos por la lectura del manuscrito; sus comentarios y sugerencias mejoraron sustancialmente la calidad de su contenido.

\section{Literatura citada}

Anónimo 1988. Compendio de estudios oceanográficos. 191 pp. Secretaría de Marina, México.

Calderón-Pérez JA, \& CR Poli. 1987. A physical approach to the postlarvae Penaeus immigration mechanism in a Mexican coastal lagoon (Crustacea: Decapoda: Penaeidae). Anales del Instituto de Ciencias del Mar y Limnología, Universidad Nacional Autónoma, México 14 (1): 147-156.

Calderón-Pérez JA, Macías-Regalado E \& S RendónRodríguez. 1989. Clave de identificación para los estadios de postlarva y primeros juveniles de camarón del género Penaeus (Crustacea: Decapoda) del Golfo de California, México. Ciencias Marinas 15 (3): 57-70.

Gallegos-García A \& J Barberán-Falcón. 1998. Surgencia eólica. En: Tapia-García M (ed), El Golfo de Tehuantepec: el ecosistema y sus recursos 3: 27-34. Universidad Autónoma Metropolitana - Iztapalapa, México.

García E. 1981. Modificaciones al sistema de clasificación climática de Köppen. 252 pp. UNAM 3ª ed, México.

García S \& L Le Reste. 1986. Ciclos vitales, dinámica, explotación y ordenación de las poblaciones de camarones peneidos costeros. FAO Documentos Técnicos de Pesca 203, 180 pp.

Gracia AG, VM Díaz-García \& LC Velásquez-Simental. 1986. Registro de una mortalidad masiva del cangrejo portunido Euphylax dovii Stimpson, 1860, en las Salinas del Marqués, Oaxaca, México. Ciencias Marinas 12 (3): 34-40.

Macías-Regalado E \& A Calderón-Pérez. 1979. Talla de inmigración de postlarvas de camarón al sistema lagunar Huizáche-Caimanero, Sinaloa, México. (Crustacea, Decapoda, Penaeus). Anales del Instituto de Ciencias del Mar y Limnología, Universidad Nacional Autónoma, México 6(2): 99-106. 
Macías-Regalado E, H Fernández-Pérez \& A CalderónPérez A. 1982. Variación diurna de la densidad de postlarvas de camarón, en la boca del sistema lagunar Huizache-Caimanero Sinaloa, México. (Crustacea: Decapoda: Penaeidae). Anales del Instituto de Ciencias del Mar y Limnología, Universidad Nacional Autónoma, México 9 (1) 381-386.

Mair JM. 1979. The identification of postlarvae of four species of Penaeus (Crustacea: Decapoda) from the Pacific coast of Mexico. Journal of Zoology 188: 347-351.

Mariano AAM. 1994. Incidencia postlarval de tres especies de camarón del género Penaeus ( $P$. vannamei, Boone, 1931; P. stylirostris, Stimpson, 1874 y P. californiensis, Holmes, 1900), Crustacea: Decapoda, en un ciclo anual en la boca-barra de Tonalá, Chiapas, México. Tesis de Licenciatura, Facultad de Biología, Universidad Veracruzana, Tuxpan, Veracruz, México, 58 pp.

Mason RD \& DA Lind. 1998. Estadística para administración y economía, 1056 pp. Editorial Alfa Omega.

Medina-Reyna CE. 1999. La retención de las artes de pesca de camarón en la laguna Mar Muerto de Oaxaca. México. Ciencia y Mar 3 (7): 13-22.

Medina-Reyna J \& HT Salinas O. 1996. Abundancia relativa de postlarvas de Penaeus spp, en el sublitoral costero de la bahía La Ventosa, Salina Cruz, Oaxaca, durante el primer semestre de 1996. Informe Final de Servicio Social. Centro Regional de Investigación Pesquera Salina Cruz, Oaxaca. Instituto Nacional de Pesca, México, 49 pp.
Monreal-Gómez MA \& DA Salas de León. 1998. Dinámica y estructura termohalina. En: M Tapia-García (ed), El Golfo de Tehuantepec: el ecosistema y sus recursos 2: 1326. Universidad Autónoma Metropolitana-Iztapalapa, México.

Pérez PAM, IE Reyna C, HT Salinas O, S Sarmiento N \& HA Gil L. 1991. Resultados del estudio sobre el recurso camarón (Penaeus sp.) en el sistema lagunar Mar Muerto, Oaxaca-Chiapas. Informe final de Investigación. Centro Regional de Investigación Pesquera Salina Cruz, Oaxaca. Instituto Nacional de Pesca, México, 15 pp.

Sánchez-Meráz B \& JA Martínez-Vega. 2000. Inmigración de postlarvas de camarón Litopenaeus sp. y Farfantepenaeus sp. a través de la Boca El Oro del Sistema Lagunar Corralero-Alotengo, Oaxaca. México. Ciencia y Mar 4 (12): 29-46.

Webb S \& RT Kneib. 2004. Individual growth rates and movement of juvenile white shrimp (Litopenaeus setiferus) in a tidal marsh nursery. Fishery Bulletin 102:376-388. 